\title{
Proximal tubular epithelial insulin receptor mediates high-fat diet-induced kidney injury
}

Hak Joo Lee, ${ }^{1}$ Meenalakshmi M. Mariappan, ${ }^{1}$ Luke Norton, ${ }^{2}$ Terry Bakewell, ${ }^{2}$ Denis Feliers, ${ }^{1}$ Sae Byeol Oh, ${ }^{1}$ Andrew Donati, ${ }^{1}$ Cherubina S. Rubannelsonkumar, ${ }^{1}$ Manjeri A. Venkatachalam, ${ }^{3}$ Stephen E. Harris, ${ }^{4}$ Isabelle Rubera, ${ }^{5}$ Michel Tauc, ${ }^{5}$ Goutam Ghosh Choudhury, ${ }^{1,6,7}$ C. Ronald Kahn, ${ }^{8}$ Kumar Sharma, ${ }^{1,6}$ Ralph A. DeFronzo, ${ }^{2}$ and Balakuntalam S. Kasinath ${ }^{1,6,7}$

${ }^{1}$ Center for Renal Medicine, Division of Nephrology, ${ }^{2}$ Division of Diabetes, Department of Medicine, ${ }^{3}$ Department of Pathology, and ${ }^{4}$ Department of Periodontics, University of Texas Health, San Antonio, Texas, USA. 'Universite Cote d'Azur, CNRS - UMR-7370, Laboratoire de Physiomédecine Moléculaire, Nice, France. ${ }^{6}$ VA Research and 7 Ceriatric Research, Education and Clinical Center, South Texas Veterans Health Care System, San Antonio, Texas, USA. ${ }^{8}$ Joslin Diabetes Center and Harvard Medical School, Boston, Massachusetts, USA.

The role of insulin receptor (IR) activated by hyperinsulinemia in obesity-induced kidney injury is not well understood. We hypothesized that activation of kidney proximal tubule epithelial IR contributes to obesity-induced kidney injury. We administered normal-fat diet (NFD) or high-fat diet (HFD) to control and kidney proximal tubule IR-knockout (KPTIRKO) mice for 4 months. Renal cortical IR expression was decreased by $60 \%$ in male and female KPTIRKO mice. Baseline serum glucose, serum creatinine, and the ratio of urinary albumin to creatinine (ACR) were similar in KPTIRKO mice compared to those of controls. On HFD, weight gain and increase in serum cholesterol were similar in control and KPTIRKO mice; blood glucose did not change. HFD increased the following parameters in the male control mice: renal cortical contents of phosphorylated IR and Akt, matrix proteins, urinary ACR, urinary kidney injury molecule-1-to-creatinine ratio, and systolic blood pressure. Renal cortical generation of hydrogen sulfide was reduced in HFD-fed male control mice. All of these parameters were ameliorated in male KPTIRKO mice. Interestingly, female mice were resistant to HFD-induced kidney injury in both genotypes. We conclude that HFD-induced kidney injury requires renal proximal tubule IR activation in male mice.

Conflict of interest: KS is on the data safety monitoring boards of Sanofi and Cara Therapeutics. $R A D$ is on the advisory boards of AstraZeneca, Novo Nordisk, Janssen, Boehringer-Ingelheim, and Intarcia. He receives research grant support as an investigator from BoehringerIngelheim, AstraZeneca, Janssen, and Merck and is on the speakers' bureaus of Novo Nordisk and AstraZeneca, for which he receives honoraria.

Copyright: (c) 2021, Lee et al. This is an open access article published under the terms of the Creative Commons Attribution 4.0 International License.

Submitted: September 14, 2020 Accepted: December 29, 2020 Published: February 8, 2021

Reference information: JCI Insight. 2021;6(3):e143619.

https://doi.org/10.1172/jci.

insight.143619.

\section{Introduction}

Insulin-resistant states, such as obesity, diabetes, and hypertension, are common causes of chronic kidney disease (CKD, refs. 1-3). Obesity predisposes to type 2 diabetes. Data from the CDC indicate that the prevalence of obesity in the US adult population is $39.8 \%$, affecting nearly 93 million people, whereas it is $18.5 \%$ in youth (4). If the same trend continues, by 2030 nearly half of all adults will be obese (5).

Obesity causes kidney injury, leading to albuminuria, glomerular enlargement, focal and segmental glomerulosclerosis, and interstitial fibrosis (6). Compared with healthy adults, subjects with obesity carry a 2.8fold and 6.3-fold higher risk for $\mathrm{CKD}$ and proteinuria, respectively, after adjusting for creatinine, uric acid, hypertension, HDL-cholesterol, and hyperglycemia (7). Studies on mechanisms through which obesity affects the kidney have assigned a role for dyslipidemia, adiponectin, angiotensin II, oxidative stress, hyperfiltration, immune activation, and lipotoxicity (8-14). In high-fat diet-induced (HFD-induced) obesity inhibition of AMP-activated protein kinase (AMPK) contributes to dyslipidemia, activation of $\mathrm{Na}-\mathrm{K}-2 \mathrm{Cl}$ cotransporter, and hypertension $(9,15,16)$. However, the critical events proximal to these pathways are not known. For instance, whether hyperinsulinemia, a key feature in obesity, plays a pathogenic role has not been directly examined.

Our suspicion that hyperinsulinemia could be involved in renal injury arose when we observed that renal cortical laminin accumulation occurred by nontranscriptional mechanisms in $d b / d b$ mice with hyperinsulinemia, obesity, and type 2 diabetes (17). These nontranscriptional mechanisms could involve stimulation of mRNA translation of ambient laminin transcripts by increased levels of insulin, a powerful inducer of that ribosomal process. First, we established that insulin receptor (IR) was activated in the renal cortex 
of $d b / d b$ mice (18). Second, studies in proximal tubular epithelial cells in vitro indicated that insulin rapidly drove laminin mRNA translation similar to high glucose (19-21). Finally, institution of a combined high-glucose and high-insulin clamp for 7 hours in normal rats reproduced mRNA translation pathways leading to laminin accumulation (22). This study left unanswered the question of the role of hyperinsulinemia independent of hyperglycemia in kidney injury. Thus, we employed the HFD model in this study, with hyperinsulinemia as the variable in the absence of hyperglycemia.

The goal of the present study was to test the hypothesis that renal proximal tubule epithelial IR activation contributes to obesity-induced kidney injury. We used kidney proximal tubule IR-knockout (KPTIRKO) mice with targeted deletion of the IR in the proximal tubule epithelial cell. Our findings reveal that activation of proximal tubular IR is required for obesity-induced kidney injury in mice.

\section{Results}

Characterization of KPTIRKO mice. KPTIRKO mouse progeny grew normally. At 3 months of age, body weight and kidney weight of KPTIRKO mice were similar to those of the control mice (Supplemental Figure 1; supplemental material available online with this article; https://doi.org/10.1172/jci. insight.143619DS1).

Immunoblotting showed that IR $\beta$ chain expression was reduced by approximately $60 \%$ in the renal cortexes of both male and female KPTIRKO mice (Figure 1, A and B). The residual expression of IR $\beta$ in the renal cortex is probably due to unaltered expression in the glomeruli, ascending limb of loops of Henle, distal tubules, and collecting ducts. Additionally, because Sglt2 is expressed mostly in the S1 segment of the proximal tubules (23), it is likely that IR is intact in the S2 and S3 segments (24). IR $\beta$ expression in KPTIRKO mice was unchanged in the liver, skeletal muscle, heart, brain, and pancreas (Supplemental Figure 2) as well as the lung and spleen (data not shown). There was no change in the IGF-I receptor (IGF-IR) expression in the kidney cortexes of male and female KPTIRKO mice (Supplemental Figure 3), indicating that there was no compensatory increase in IGF-IR expression for the loss of IR $\beta$. Survey of signaling kinases showed no change in the phosphorylation of Akt or Erk in the renal cortexes of KPTIRKO mice compared with that in control mice (Supplemental Figure 3). There was no difference in the expression of renal cortical content of matrix proteins laminin or fibronectin in the KPTIRKO mice relative to that in control mice (data not shown). Serum chemistries showed no changes in blood urea nitrogen and serum creatinine (Supplemental Table 1).

Effect of HFD on food consumption and body weight. Control and KPTIRKO mice were randomized to receive normal-fat diet (NFD) or HFD for 4 months and underwent monthly evaluation, as shown in Figure $1 \mathrm{C}$. There was no difference in body weight at baseline before randomization to NFD versus HFD among mice assigned to the 4 groups (control mice on NFD, control mice on HFD, KPTIRKO mice on NFD, and KPTIRKO mice on HFD). HFD resulted in weight gain to an equal extent in male control and KPTIRKO mice (Supplemental Figure 4). Similarly, female control and KPTIRKO mice also gained comparable weight on HFD (Supplemental Figure 4). Food consumption measurement showed that it was higher initially in HFD-fed control and KPTIRKO mice relative to NFD fed mice in both sexes; however, food consumption decreased in HFD-fed mice from that time point comparable to NFD fed mice, despite continued weight gain (Supplemental Figure 4).

HFD-induced albuminuria and tubular injury are reduced in KPTIRKO mice. There was no difference in the urinary albumin-to-creatinine ratio (ACR) between male and female control and KPTIRKO mice on NFD at baseline and for the duration of the study. In male control mice, HFD increased the urinary ACR at 2, 3, and 4 months. In KPTIRKO mice, HFD increased ACR only at 2 and 3 months, and from the second month, ACR was significantly lower in KPTIRKO mice relative to control mice until the end of the study (Figure 1D). Over the 4-month period of HFD, control mice had a significantly longer duration of albuminuria compared with HFD-fed KPTIRKO mice. In female mice, HFD increased ACR, which was significantly lower in the KPTIRKO mice, only at the second month time point (Figure 1E).

Renal cortical nephrin content was unchanged among the HFD-fed groups compared with NFDfed groups, suggesting that albuminuria was likely not due to podocyte injury (Supplemental Figure 5). Increased urinary kidney injury molecule-1 (KIM-1) excretion is an index of proximal tubular injury (25). The ratio of urinary KIM-1 to creatinine and renal cortical KIM-1 content were increased in HFD-fed male control mice but not in KPTIRKO mice (Figure 1, F and H); these parameters did not change in HFD-fed female mice of both genotypes (Figure 1, G and I). These observations show the 
A

Male
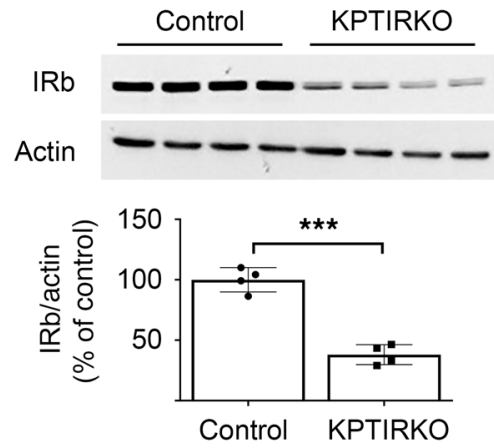

D

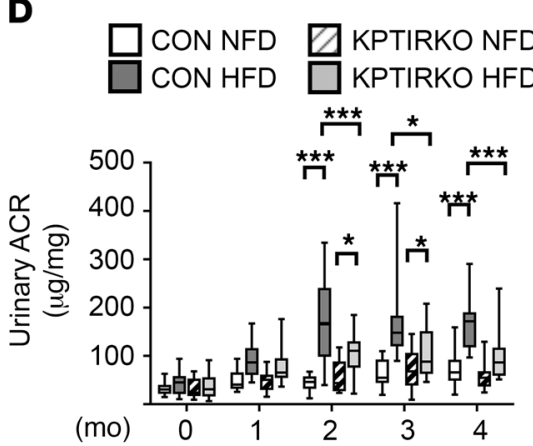

$\mathbf{F}$

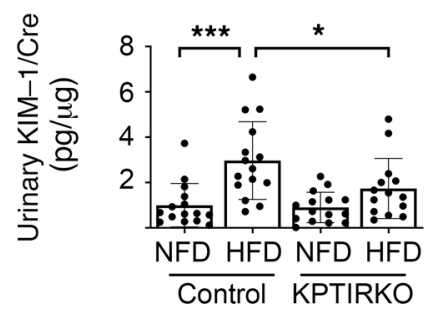

H

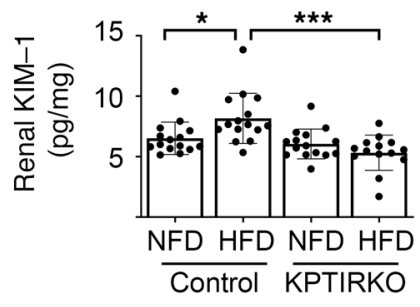

B

Female
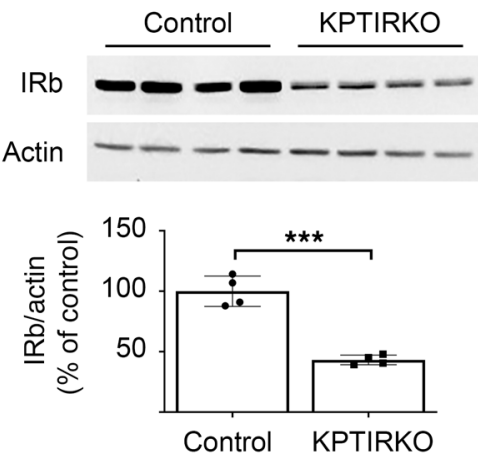

E
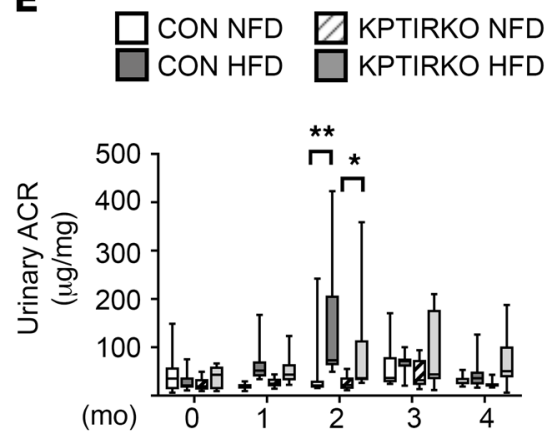

G

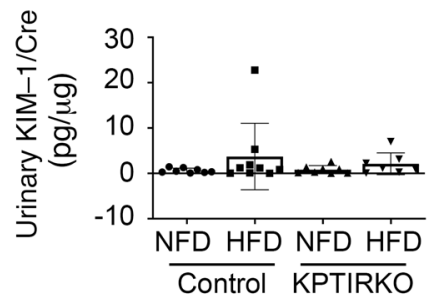

I

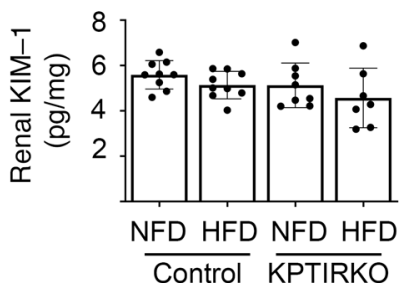

C

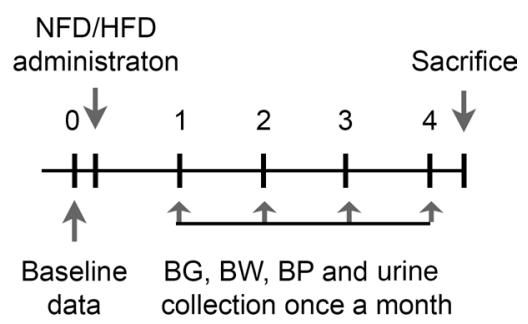

Figure 1. KPTIRKO mice are resistant to HFD-induced kidney injury. (A and B) Immunoblotting showed significantly reduced renal cortical IR expression in male and female mice, respectively (4 mice in each group, ${ }^{* *} P<0.001$ by $t$ test). (C) Schematic of administration of NFD or HFD and data collection. Blood glucose (BC), body weight (BW), blood pressure (BP) were measured and urine was collected for analysis once per month (14-15 male mice per group, 7-9 mice female mice per group). (D and E) HFD-induced albuminuria, measured as the urinary albumin-to-creatinine ratio (ACR), was inhibited in KPTIRKO male ( $n=14-15$ per group) and female $\left(n=7-9\right.$ per group) mice $\left({ }^{*} P<0.05,{ }^{* *} P<0.01,{ }^{* * *} P<0.001\right.$ by 2 -way ANOVA). (F and $\mathbf{G})$ HFD increased the urinary KIM-1-to-creatinine ratio in male control mice but not in KPTIRKO mice ( $n=14-15$ per group) (F); it was unaffected in female mice (G) ( $n=7-8$ per group) $\left({ }^{*} P<0.05\right.$, ${ }^{* *} P<0.001$ by 1 -way ANOVA and post hoc analysis using Tukey's multiple-comparisons test). (H and I) HFD led to an increase in renal cortical KIM-1 expression in male control but not KPTIRKO mice ( $n=14-15$ per group); HFD did not affect KIM-1 expression in female mice of either genotype ( $n=7-9$ per group) ${ }^{*} P<0.05,{ }^{* *} P<0.001$ by 1 -way ANOVA and post hoc analysis using Tukey's multiple-comparisons test). 
following: (a) HFD-induced albuminuria is dependent on proximal tubule IR in male and female mice. (b) HFD-induced albuminuria is likely due to proximal tubular injury. (c) HFD-induced albuminuria is milder in female mice, which resist HFD-induced proximal tubular injury.

HFD-induced hypertension is ameliorated in male KPTIRKO mice. HFD was associated with a sustained increase in systolic blood pressure in male control mice for the duration of the study. Male KPTIRKO mice demonstrated a lower systolic blood pressure at nearly all time points (Figure 2A). There was no significant difference between systolic blood pressures of male control and KPTIRKO mice on NFD at most time points. The heart-to-tibia ratio was increased in HFD-fed male control mice with hypertension consistent with cardiac hypertrophy; this did not occur in HFD-fed male KPTIRKO mice, suggesting absence of cardiac hypertrophy, possibly related to lack of hypertension (Figure 2C). These data agree with those in the report by Wang et al. (26). Systolic blood pressure generally did not exceed $140 \mathrm{~mm} \mathrm{Hg}$ on either diet in female mice of either genotype (Figure 2B); correspondingly, there was no change in the heart-to-tibia ratio in female mice fed HFD (Figure 2D). Thus, male but not female mice are susceptible to HFD-induced hypertension and heart hypertrophy, which requires the presence of IR in the proximal tubule.

HFD-induced renal matrix changes are reduced in male KPTIRKO mice. The kidney-to-tibia ratio was increased in male control mice but not in male KPTIRKO mice fed HFD (Figure 3A), suggesting that IR is required for kidney hypertrophy to occur. Interestingly, HFD induced kidney hypertrophy in both genotypes in female mice (Figure 3B), suggesting that either IR in other tubular segments and/or other growth regulators may be sufficient to induce HFD-induced kidney hypertrophy. We examined the levels of matrix proteins that contribute to renal fibrosis. Immunoblotting showed increased renal cortical content of laminin $\gamma 1$, fibronectin, and collagen I $\alpha 2$ in male control mice fed HFD but not in male KPTIRKO mice (Figure $3, \mathrm{C}, \mathrm{E}$, and G). There was no change in the expression of these matrix proteins in female mice fed HFD (Figure 3, D, F, and H). These data demonstrate that (a) in male mice proximal tubular IR is required for kidney hypertrophy and increase in renal cortical matrix proteins following HFD and (b) that female mice resist HFD-induced matrix protein increase in the renal cortex, although they manifest renal hypertrophy.

HFD-induced signaling pathways are unchanged in male KPTIRKO mice. Insulin is a powerful inducer of protein synthesis via regulation at the level of mRNA translation in kidney cells $(19,21)$. Hyperinsulinemia could stimulate the synthesis of matrix proteins in HFD-induced kidney injury. We examined signaling events related to IR activation that are linked to protein synthesis. IR signaling begins with phosphorylation at key tyrosine residues 1150 and 1151 in the IR $\beta$ chain (27). Renal cortical IR tyrosine phosphorylation at Tyr1150/1151 was increased in HFD-fed male control mice but not in KPTIRKO mice (Figure 4A). HFD did not result in changes in IR Tyr-1150/1151 phosphorylation in female mice of either genotype (Figure 4B). On NFD, fasting serum insulin levels were similar in male control and KPTIRKO mice; HFD resulted in hyperinsulinemia in both control and KPTIRKO mice to the same extent (Supplemental Figure 6). There was no difference in random serum insulin levels among the 4 groups of female mice (Supplemental Figure 6). IR activation leads to activation of Akt, which controls downstream signaling events leading to protein synthesis $(28,29)$. We assessed Akt activity by examining the phosphorylation of glycogen synthase kinase $3 \beta$ (GSK3 3 ), a direct substrate of Akt. Phosphorylation of GSK3 $\beta$ by Akt at Ser9 leads to inhibition of its activity and removes inhibition on protein synthesis $(30,31)$, including in kidney cells (32). Conversely, stimulation of GSK3 $\beta$ inhibits synthesis of proteins in the kidney (33). HFD increased the renal cortical GSK3 $\beta$ phosphorylation at Ser9 in male control mice but not in KPTIRKO mice (Figure 4C). HFD did not affect GSK3 $\beta$ phosphorylation in female control or KPTIRKO mice (Figure 4D). Akt-induced inhibition of GSK3 $\beta$ reduces Ser535 phosphorylation of its direct substrate, eukaryotic initiation factor $2 \mathrm{~B}$ (eIF2B $\varepsilon$, refs. 32,34 ). eIF2B is a heteropentamer and its $\varepsilon$ component promotes GDP/GTP exchange on eIF2, a key regulatory step in the initiation phase of mRNA translation (35). HFD reduced eIF2B $\varepsilon$ phosphorylation in the renal cortexes of male control mice consistent with decreased GSK3 $\beta$ activity but not in HFD-fed KPTIRKO mice (Figure 4E). There was no change in eIF2Be phosphorylation in female mice on HFD (Figure 4F), in alignment with unchanged activity of GSK3 $\beta$.

Downstream of Akt, mTORC1 governs mRNA translation by directly phosphorylating p70S6 kinase and eukaryotic initiation factor $4 \mathrm{E}$ binding protein (29). Akt activates mTORC1 in several ways. It phosphorylates tuberous sclerosis 1 (TSC-1) and AMPK, leading to activation of Rheb, which promotes mTORC1 activity (29). Additionally, it phosphorylates PRAS40, which is a tonic inhibitor of mTORC1. Activation of mTORC1 involves Ser2448 phosphorylation. HFD promoted mTOR phosphorylation at this site in male control mice but not in male KPTIRKO mice (Figure 5A). HFD did not affect mTORC1 
A
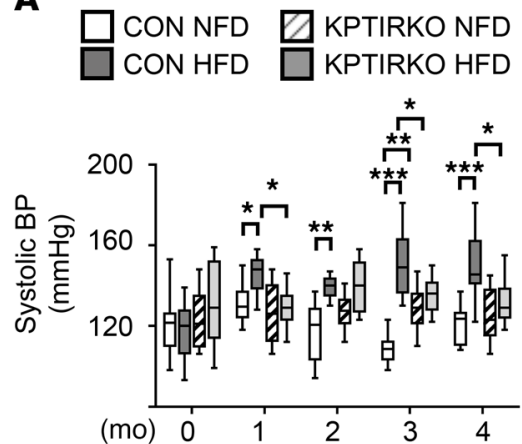

C

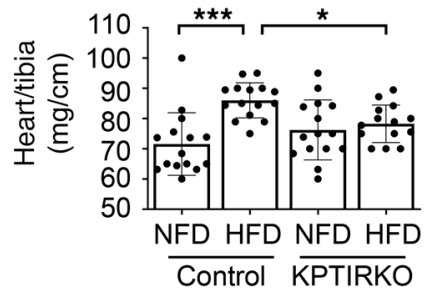

B
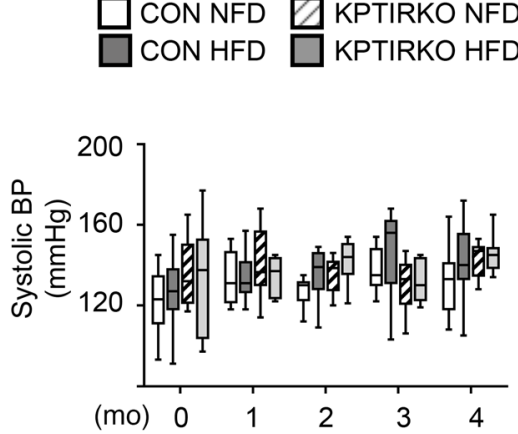

D

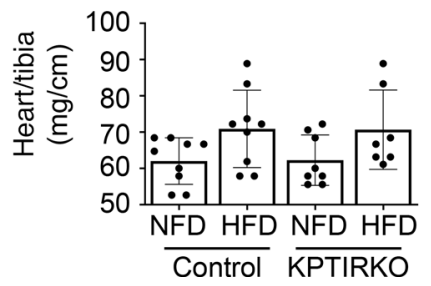

Figure 2. HFD-induced hypertension and heart hypertrophy are mitigated in KPTIRKO mice. (A and B) Systolic blood pressure (BP) was increased on HFD in male control mice but not KPTIRKO mice ( $n=14-15$ per group). It was unchanged by HFD in female control and KPTIRKO mice ( $n=7-9$ per group) $\left({ }^{*} P<0.05,{ }^{*} P<0.01,{ }^{* * *} P<0.001\right.$ by 2 -way ANOVA and post hoc analysis using Tukey's multiple-comparisons test). (C and $\mathbf{D})$ HFD led to an increase in the heart weightto-tibia ratio in male control mice but not in KPTIRKO mice ( $n=14-15$ per group); the ratio was unchanged in female mice on HFD ( $n=7-9$ per group) $\left({ }^{*} P<0.05,{ }^{* * *} P<0.001\right.$ by 1 -way ANOVA and post hoc analysis using Tukey's multiple-comparisons test). In the graphs, each dot represents 1 mouse. Data are presented as mean \pm SD.

phosphorylation in female mice of either genotype (Figure 5B). Renal cortical p70S6 kinase phosphorylation was increased in male mice fed HFD but not in KPTIRKO mice on the same diet (Figure 5C); it was unchanged among the 4 groups of female mice (Figure 5D). Activation of mTORC1-p70S6 kinase regulates mRNA translation, a rate-limiting step in protein synthesis $(29,36)$, and is likely to be related to increase in synthesis of proteins, including matrix proteins, in HFD-fed mice.

The energy sensor, AMPK, is a major regulator of protein synthesis in the kidney; its activity is inhibited in renal injury associated with diabetes $(10,37,38)$. AMPK activity is also inhibited in HFD-associated kidney injury $(9,39)$. Akt inhibits AMPK by phosphorylation (40). We examined AMPK activity by studying Ser79 phosphorylation of acetyl-CoA carboxylase (ACC), its direct substrate. HFD inhibited ACC phosphorylation in the renal cortexes of male control mice (Figure 5E). It was unchanged in HFD-fed male KPTIRKO mice compared with NFD fed mice, and, it was significantly higher compared with HFD-fed control mice (Figure $5 \mathrm{E}$ ). In female mice, ACC phosphorylation showed a trend toward increase in HFD-fed controls whereas it was unchanged in KPTIRKO mice (Figure 5F). These data collectively show the following: (a) HFD induces renal cortical IR activation in male mice, leading to stimulation of Akt and inhibition of AMPK, and, increase in the activity of mTORC1, which governs mRNA translation. We have previously shown that these events facilitate synthesis of proteins, including matrix proteins $(20,32,33)$. (b) In male mice, IR is required for stimulation of Akt, mRNA translation signaling events, and AMPK inactivation following HFD. (c) HFD does not promote IR phosphorylation and downstream signaling events in female mice. The trend toward increased AMPK activity in female control HFD may represent a defense against stimulation of protein synthesis events by HFD.

HFD reduces hydrogen sulfide generation only in male control but not KPTIRKO mice. Hydrogen sulfide $\left(\mathrm{H}_{2} \mathrm{~S}\right)$, a gasotransmitter, is constitutionally synthesized by the kidney. It regulates glomerular filtration rate, renal blood flow, and tubular handling of electrolytes (41). $\mathrm{H}_{2} \mathrm{~S}$ is generated in the kidney in cytosolic and mitochondrial metabolic reactions related to L-cysteine, with the participation of cystathionine $\beta$-synthase (CBS), cystathionine $\gamma$-lyase (CSE), and 3-mercaptopyruvate sulfotransferase $(42,43)$. An additional pathway involving D-cysteine also operates in the kidney (44). We have previously reported that CBS and CSE expression, and generation of $\mathrm{H}_{2} \mathrm{~S}$, are reduced in the renal cortexes of aged mice, marmosets, and mice with type 1 and type 2 diabetes (45-47). Aging-associated albuminuria, renal fibrosis, and activation of the IR signaling pathway are also associated with $\mathrm{H}_{2} \mathrm{~S}$ deficiency in mice (45). These injury parameters 
A
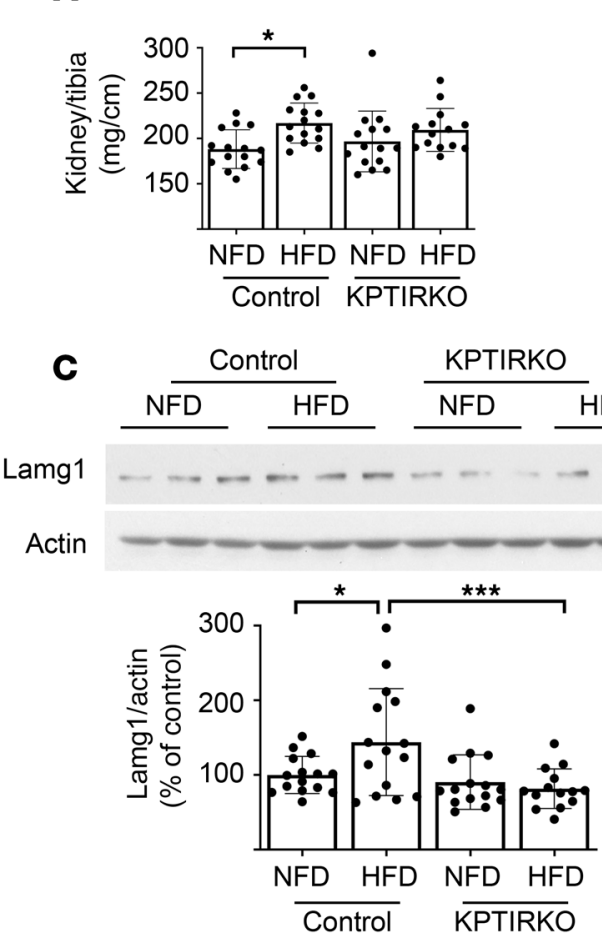

B

Female
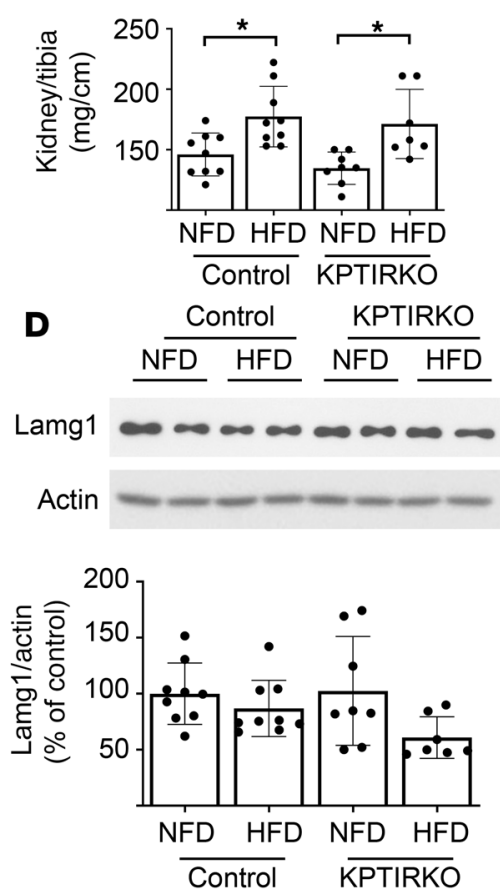

$\mathbf{F}$

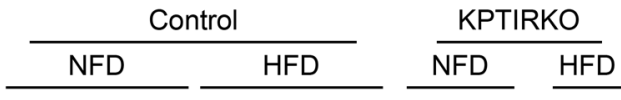

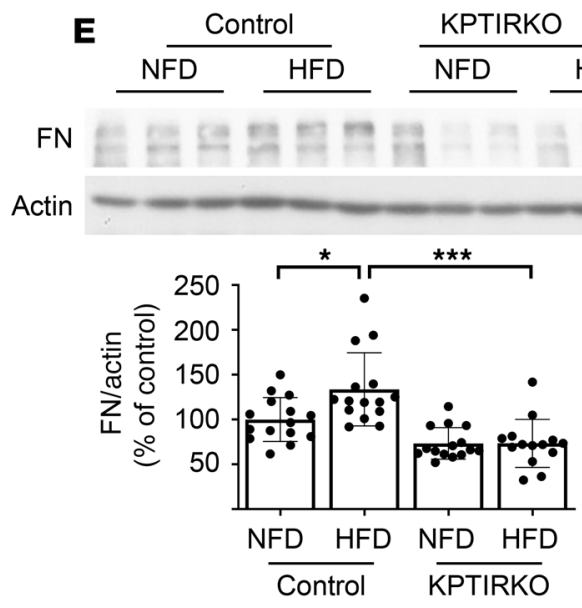

G
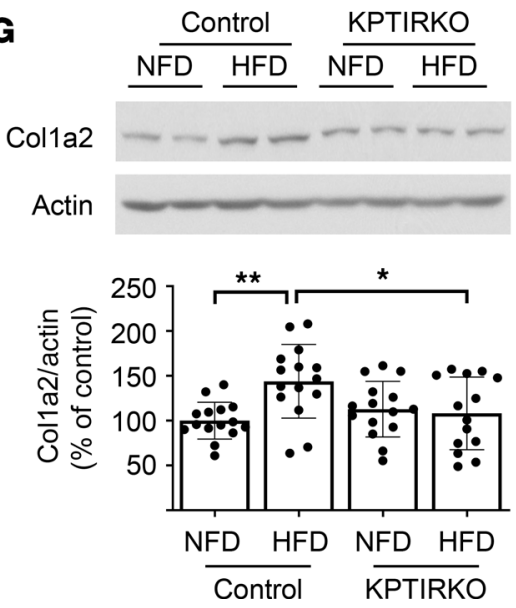

Actin

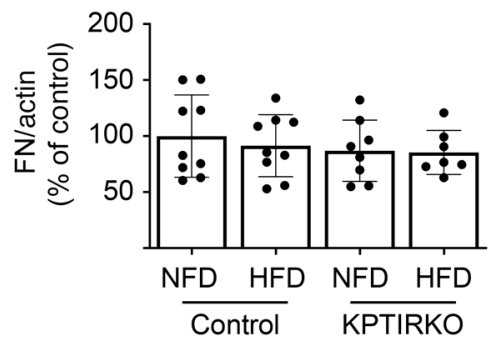

H

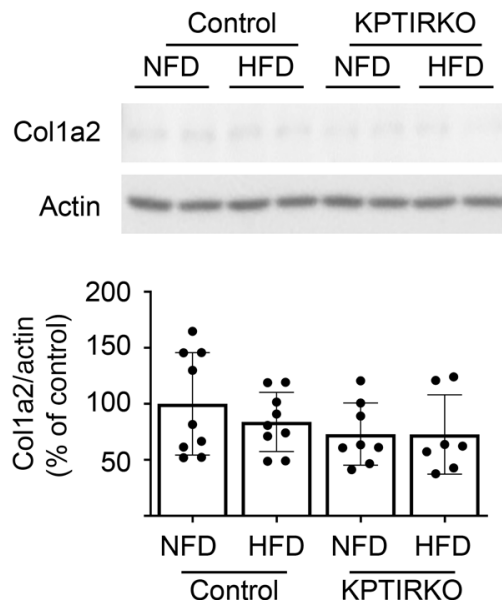

Figure 3. KPTIRKO mice are protected from matrix increase following HFD. (A and B) HFD increased the kidney-to-tibia ratio, indicating hypertrophy in male control mice but not in KPTIRKO mice ( $n=14-15$ per group). HFD increased the kidney-to-tibia ratio in female mice of both genotypes ( $n=7-9$ per group). (C-H) Immunoblotting of renal cortical extracts showed increase in the expression of laminin $\gamma 1$, fibronectin, and collagen $1 \alpha 2$ in male control but 
not in KPTIRKO mice (C, E, and G) ( $n=14-15$ per group); HFD did not affect the renal cortical content of these proteins in female mice of either genotype (D, $\mathbf{F}$, and $\mathbf{H}$ ) ( $n=7-9$ per group) ( ${ }^{*} P<0.05,{ }^{* *} P<0.01$, ${ }^{* * *} P<0.001$ by 1 -way ANOVA and post hoc analysis using Tukey's multiple-comparisons test). In the graphs, each dot represents 1 mouse. Data are presented as mean \pm SD.

in aged mice are ameliorated by the administration of sodium hydrosulfide (NaHS), a source of $\mathrm{H}_{2} \mathrm{~S}(45)$. We examined the status of $\mathrm{H}_{2} \mathrm{~S}$ in HFD-fed mice. $\mathrm{H}_{2} \mathrm{~S}$ generation by the kidney cortexes was reduced in HFD-fed male control mice but not in KPTIRKO mice (Figure 6A). In female mice, HFD did not affect $\mathrm{H}_{2} \mathrm{~S}$ generation following HFD in either genotype (Figure 6B). HFD reduced the expression of CBS but not CSE in both male control and KPTIRKO mice (Figure 6C). In female mice, CBS expression showed a trend toward reduction in HFD-fed control mice but not in KPTIRKO mice; CSE content was unaffected (Figure 6D). These data suggest the following. (a) In male mice, IR is involved in reducing $\mathrm{H}_{2} \mathrm{~S}$ generation in HFD-fed mice. (b) Maintenance of $\mathrm{H}_{2} \mathrm{~S}$ generation in HFD-fed KPTIRKO mice, despite reduced CBS expression, suggests that $\mathrm{CBS}$-independent mechanisms maintain $\mathrm{H}_{2} \mathrm{~S}$ production. (c) In female mice, $\mathrm{H}_{2} \mathrm{~S}$ generation is unchanged following HFD in control mice although CBS expression tended to decrease, indicating existence of compensatory mechanisms to maintain $\mathrm{H}_{2} \mathrm{~S}$ generation. Intact renal cortical $\mathrm{H}_{2} \mathrm{~S}$ production may aid the female mice in resisting HFD-induced kidney injury.

Metabolic studies. Random blood glucose concentration was measured once per month. In male control and KPTIRKO mice on NFD or HFD, blood glucose stayed below $8 \mathrm{mmol} / \mathrm{L}$, except for 1 spike to 8 $\mathrm{mmol} / \mathrm{L}$ at month 3 in HFD-fed control mice (Figure 7A). In female mice, blood glucose remained below $8 \mathrm{mmol} / \mathrm{L}$ in both genotypes (Figure 7B). HFD increased serum triglycerides in male and female control mice; the increase was attenuated in male KPTIRKO mice with a similar trend in female KPTIRKO mice (Figure 7, C and D). HFD increased serum cholesterol in male control and KPTIRKO mice (Figure 7E). HFD increased serum cholesterol in HFD-fed female control mice but not in KPTIRKO mice (Figure 7F). The liver-to-tibia ratio was increased in male control and KPTIRKO mice fed HFD, indicating a similar hepatic response to HFD in both genotypes (Figure 7G). This parameter was unchanged in female mice regardless of the genotype (Figure $7 \mathrm{H}$ ). These data indicate the following. (a) Serum triglyceride elevation due to HFD is lower in KPTIRKO mice compared with control mice, suggesting a possible regulatory role for proximal tubule IR in systemic triglyceride metabolism. (b) Female mice resist hepatic enlargement in HFD. Taken together our data show that the amelioration of HFD-induced kidney injury in male KPTIRKO mice is due to the absence of IR in the proximal tubules.

\section{Discussion}

Our data demonstrate that proximal tubular epithelial cell IR activation by hyperinsulinemia in HFD-fed mice is required for the development of albuminuria, hypertension, matrix protein accumulation, renal hypertrophy, dyslipidemia, and stimulation of signaling pathways involved in protein synthesis. In states of insulin resistance, the kidney may be an "innocent bystander," subject to the injurious effects of hyperinsulinemia. The increase in circulating insulin could be due to several mechanisms: (a) HFD-induced insulin resistance in extrarenal tissues; (b) direct nutrient stimulation of $\beta$ cells of the islets secondary to increased caloric intake $(48,49)$; (c) stimulation of insulin secretion by the gut-derived incretin hormones; (5) decreased metabolic clearance rate of insulin by the liver due to hepatic insulin resistance; (6) reduced renal insulin degradation due to proximal tubular IR knockout; and (7) a combination of the above.

In the kidney, IR is expressed by the constituent cells of the glomerulus and all the segments of the tubules $(50,51)$. IR knockout in specific parts of the nephron have revealed a segment- and cell-specific role of insulin in regulating physiologic functions. IR contributes to normal nephron function by preventing loss of albumin during glomerular filtration, proximal tubular maintenance of glucose homeostasis, sodium reabsorption, and defense against urinary tract infection (52-55); these effects of insulin on the kidney are achieved by selective recruitment of IR substrate 1 (IRS-1) versus IRS-2 (56). Podocyte IR-knockout mice manifest albuminuria, thickening of the glomerular basement membrane, and glomerulosclerosis mimicking diabetic injury in the presence of euglycemia (52). Under physiologic conditions, the minute amounts of albumin that pass the glomerular filtration barrier are reabsorbed by the proximal tubules mainly by the megalin-cubilin system, which is stimulated by insulin (57). Thus, absence of IR in proximal tubules should lead to albuminuria. However, in our study the basal urinary albumin excretion in KPTIRKO mice was similar that of control mice on NFD, indicating that IR-independent mechanisms compensate to conserve albumin endocytosis. Albuminuria seen in HFD-fed 
A

Male
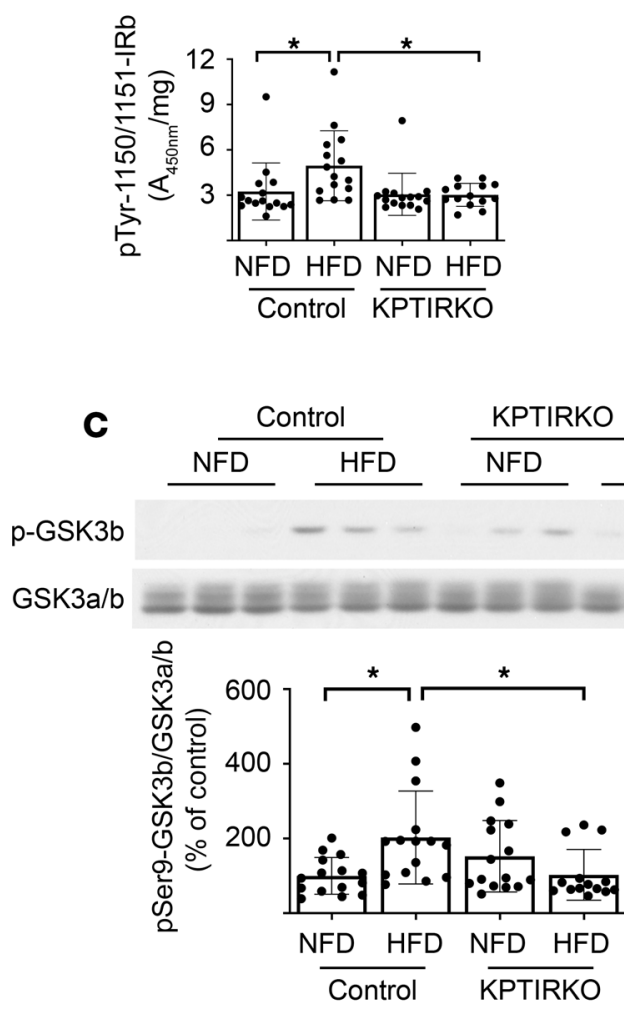

E
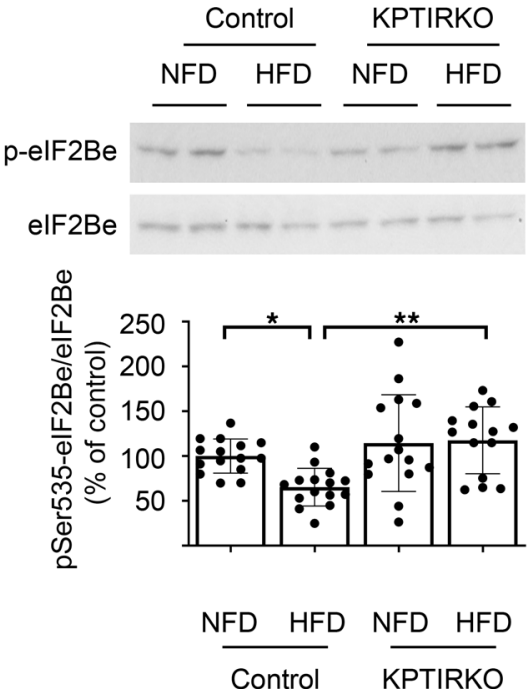

B

Female

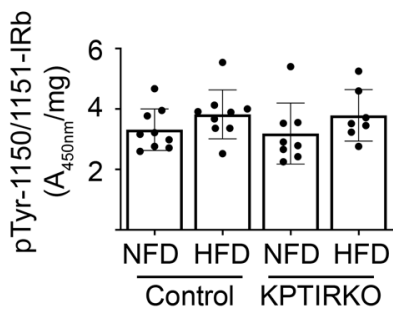

p-GSK3b

GSK3a/b
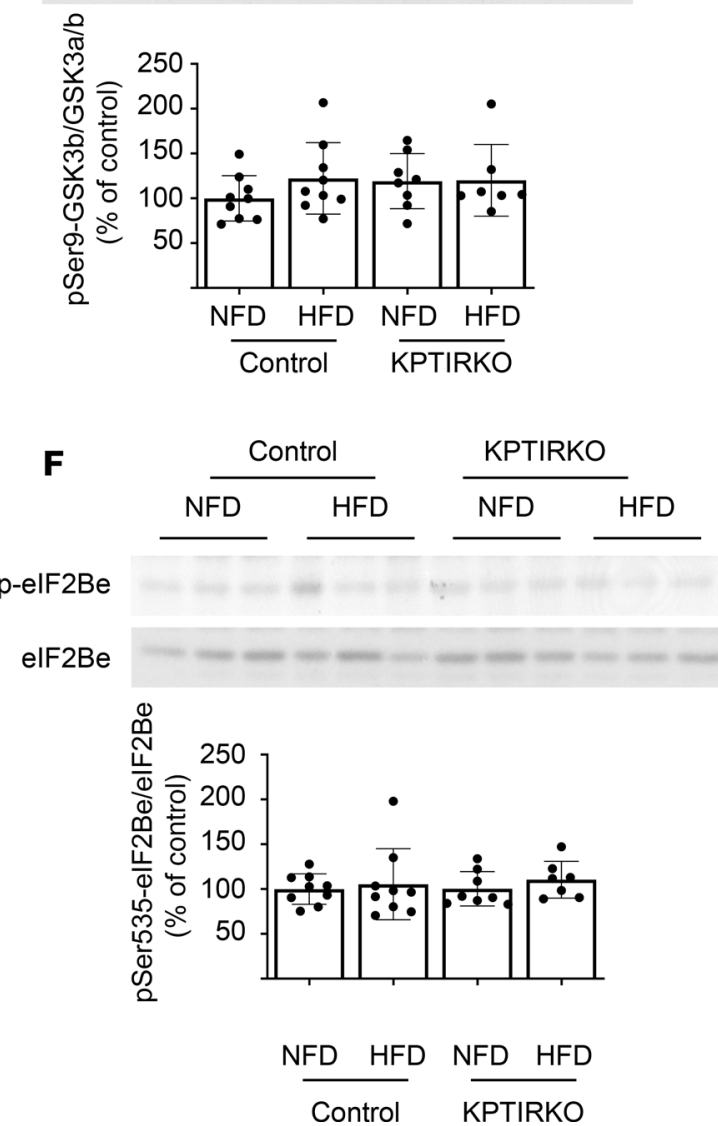

Figure 4. Signaling studies-1. (A and B) ELISA was performed to assess IR Tyr-1150/1151 phosphorylation. HFD increased this parameter in the renal cortexes of male control mice but not in KPTIRKO mice ( $n=14-15$ per group). HFD did not affect it in female mice of either genotype ( $n=7-9$ per group). (C and $\mathbf{D}$ ) HFD increased renal cortical Ser-9 phosphorylation of GSK3 $\beta$, indicating Akt activation in male control but not KPTIRKO mice ( $n=14-15$ per group); it was unchanged in female mice ( $n=7-9$ per group). (E and F) Kidney cortical phosphorylation of elF2B $\varepsilon$, an index of GSK3 $\beta$ activity, was decreased in HFD-fed male control mice but not in KPTIRKO mice ( $n=14-15$ per group); it remained unaltered in female control and KPTIRKO mice $\left(n=7-9\right.$ per group) $\left({ }^{*} P<0.05\right.$, ${ }^{* *} P<0.01$, by 1-way ANOVA and post hoc analysis using Tukey's multiple-comparisons test).

control mice was absent in KPTIRKO mice, suggesting that the proximal tubule IR mediates obesity-induced urinary protein losses. Normal nephrin expression and an increase in urinary and renal cortical KIM-1, an index of proximal tubular injury, further support the notion that HFD-induced albuminuria in control mice is due to injury to the proximal tubules rather than podocytes; absence of changes in KIM-1 in KPTIRKO mice on HFD indicates that IR mediates proximal tubular injury. 
A
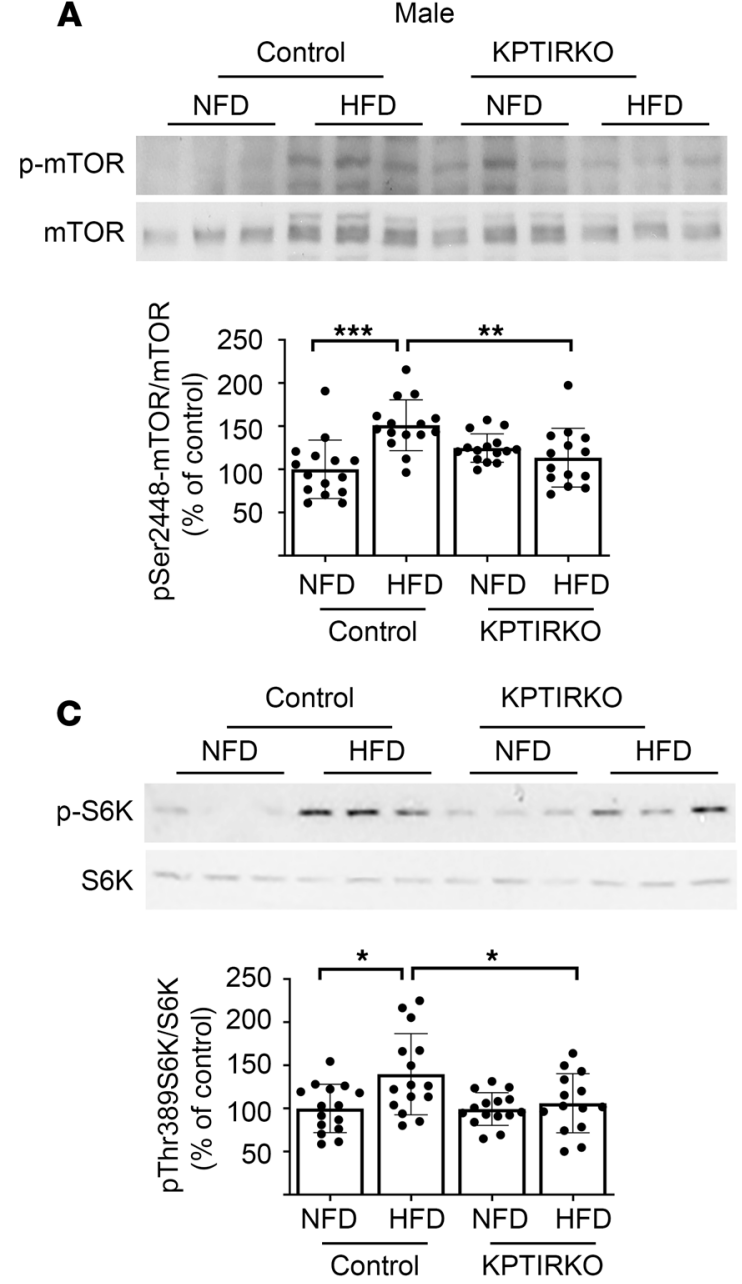

$\mathbf{E}$

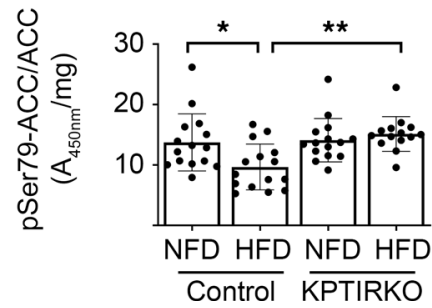

B
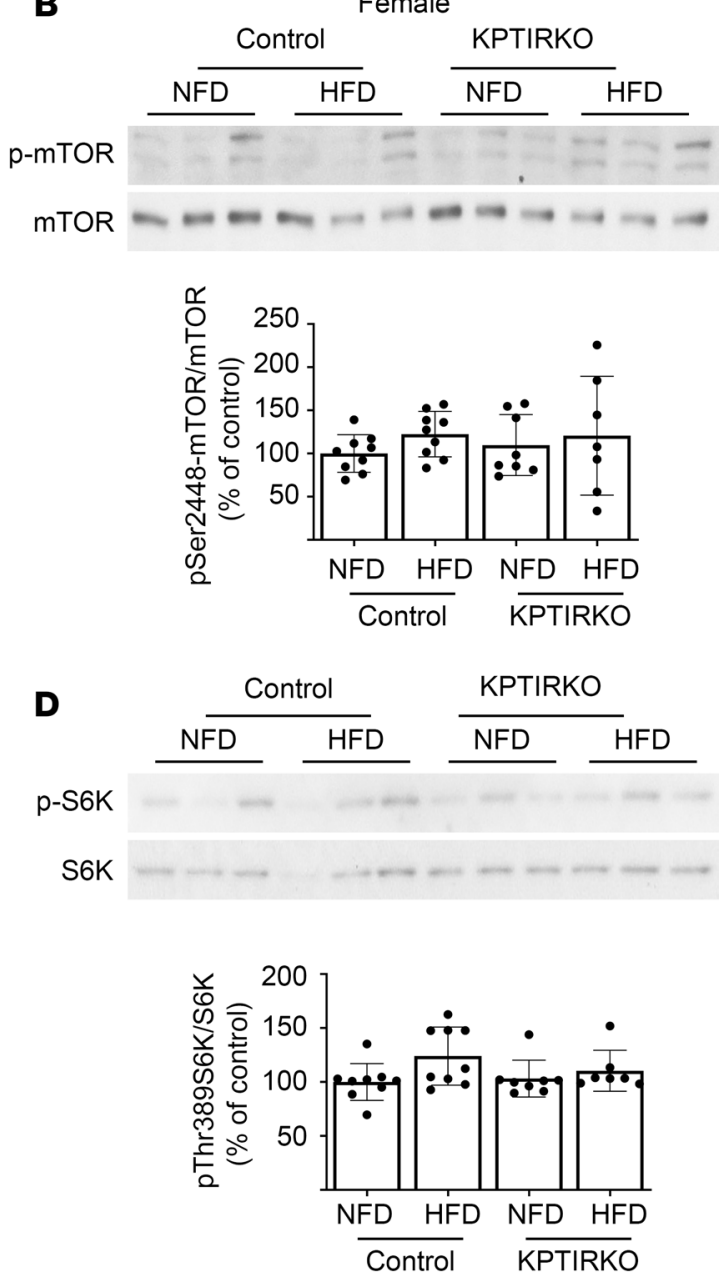

$\mathbf{F}$

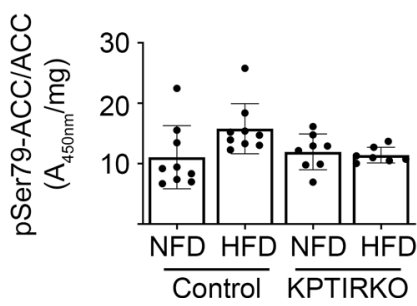

Figure 5. Signaling studies-2. (A-D) Immunoblotting showed that Ser-2448 phosphorylation of mTOR and Thr-389 phosphorylation of p70S6 kinase were increased, indicating mTORC1 activation in the renal cortexes of HFD-fed male control but not KPTIRKO mice ( $n=14-15$ per group). HFD did not affect these parameters in female mice ( $n=7-9$ per group). (E and F) Ser-79 phosphorylation of ACC was measured by ELISA as an index of AMPK activity. HFD inhibited AMPK in male control mice but not in KPTIRKO mice ( $n=14-15$ per group); it was unchanged in female mice of both genotypes ( $n=7-9$ per group) $\left({ }^{*} P<0.05,{ }^{*} P<0.01,{ }^{* *} P<0.001\right.$ by 1 -way ANOVA and post hoc analysis using Tukey's multiple-comparisons test). In the graphs, each dot represents 1 mouse. Data are presented as mean \pm SD.

The kidney is an important site of glucose production by the process of gluconeogenesis and contributes to about $20 \%$ of endogenous glucose production under postabsorptive conditions (58). Hyperglycemia has been reported in Ggt-Cre-mediated proximal tubular IR-knockout mice due to stimulation of gluconeogenesis (53). In our study, blood glucose levels monitored every month did not reveal sustained hyperglycemia in NFD-fed KPTIRKO mice; further, even the mild elevation in blood glucose seen in HFD-fed control and KPTIRKO mice was within the normal range. Possible explanations for the difference between the 2 studies could be as follows. (a) In our study, IR was absent only in the S1 segment of the proximal tubule, whereas the IR deletion may have been more extensive in 
A

Male
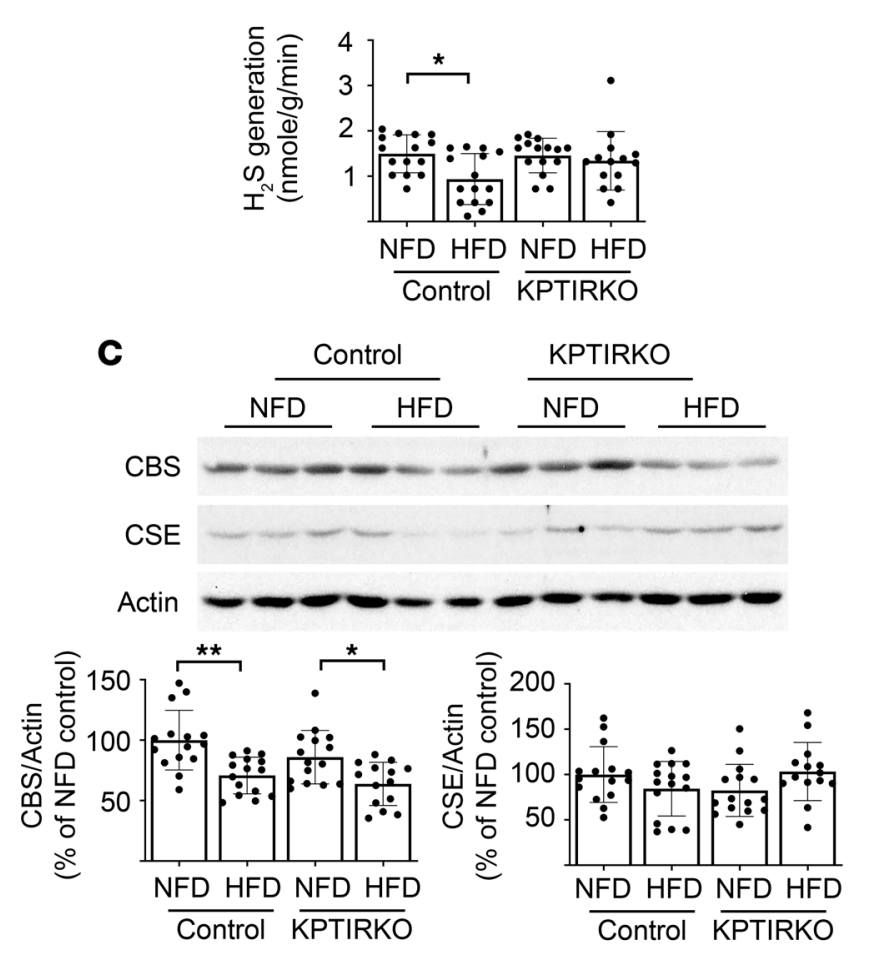

B

Female

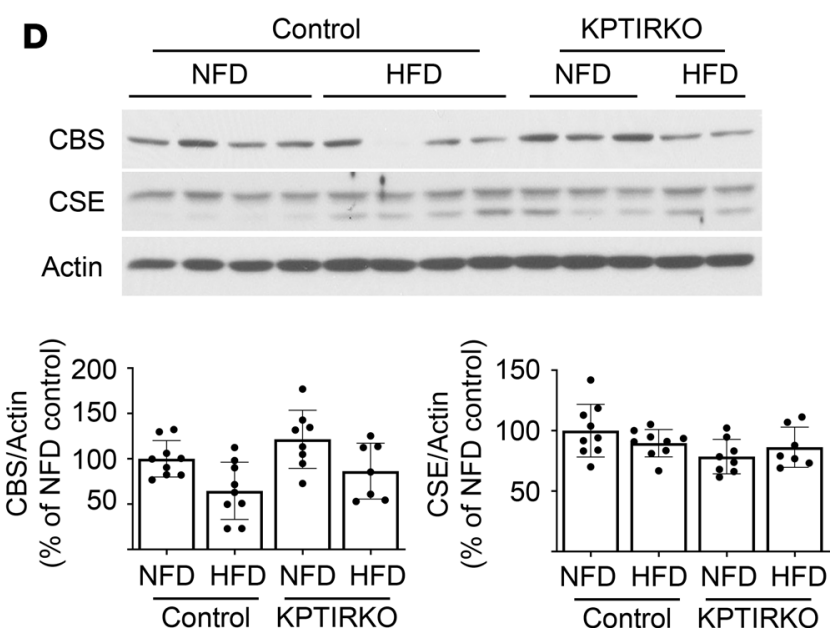

Figure 6. HFD regulation of hydrogen sulfide in the kidney. (A and B) HFD inhibited renal cortical hydrogen sulfide $\left(\mathrm{H}_{2} \mathrm{~S}\right)$ generation in male control but not KPTIRKO mice ( $n=14-15$ per group); HFD did not change $\mathrm{H}_{2} \mathrm{~S}$ generation in female control or KPTIRKO mice ( $n=7-9$ per group). (C and $\left.\mathbf{D}\right)$ Immunoblotting showed that HFD reduced the expression of cystathionine $\beta$-synthase (CBS) but not cystathionine $\gamma$-lyase (CSE) in male control and KPTIRKO mice ( $n=14-15$ per group). HFD did not affect the expression of these enzymes in female mice of either genotype ( $n=7-9$ per group) $\left({ }^{*} P<0.05\right.$, ${ }^{* *} P<0.01$ by 1 -way ANOVA and post hoc analysis using Tukey's multiple-comparisons test). In the graphs, each dot represents 1 mouse. Data are presented as mean \pm SD.

the study by Tiwari et al. (53). (b) Differences in the genetic background of mice may have played a role. Tiwari et al. used 129/Sv/C57BL6 mice whereas C57BL6 mice were employed in our study (53).

Since the pioneering studies of DeFronzo et al. on the effects of insulin on renal handling of sodium (55), many groups have used targeted deletion of IR in tubular segments to elucidate its role in hypertension. Kap-Cre-mediated deletion of IR, mainly involving the thick ascending limb and the collecting duct, led to an increase in systolic and diastolic blood pressures (59). Since IR was intact in the proximal tubules in this study, it is possible that it contributed to hypertension by increasing sodium reabsorption. In our study, HFD increased blood pressure in control mice but not in KPTIRKO mice, suggesting that proximal tubular IR was involved in the genesis of hypertension. Nakamura et al. have shown that insulin recruits the IRS2/Akt2/mTORC2 signaling pathway to activate the sodium bicarbonate cotransporter to promote sodium reabsorption in the proximal tubule and contribute to hypertension in metabolic syndrome $(60,61)$. IR/mTORC2 axis is also involved in the stimulation of sodium reabsorption by activation of the sodium-chloride cotransporter in the distal convoluted tubule and the epithelial sodium channel in the collecting duct, which could also contribute to hypertension (62-64). While hypertension could have mediated some aspects of HFD-induced kidney injury, our data show that genesis of hypertension requires intact IR signaling.

In hyperinsulinemic states, such as obesity, not all tissues and not all pathways are insulin resistant (65). The term insulin sensitivity and its reciprocal, resistance, generally have been employed in the context of glucose uptake by cells. However, in insulin-resistant states, events other than glucose transport, e.g., protein synthesis, may be activated in insulin-responsive tissues, such as the kidney and the vessel wall. There are examples of IR-mediated injury in other tissues. In diabetes, retinal neovascularization was reduced in vascular endothelial specific IR-knockout mice; IGF-1 receptor activation had a less important role $(66,67)$. Hyperinsulinemia is an independent risk factor for coronary artery disease $(68-$ 70). Cardiac IR signaling is stimulated in a pressure overload model of heart failure, whereas selective 
A
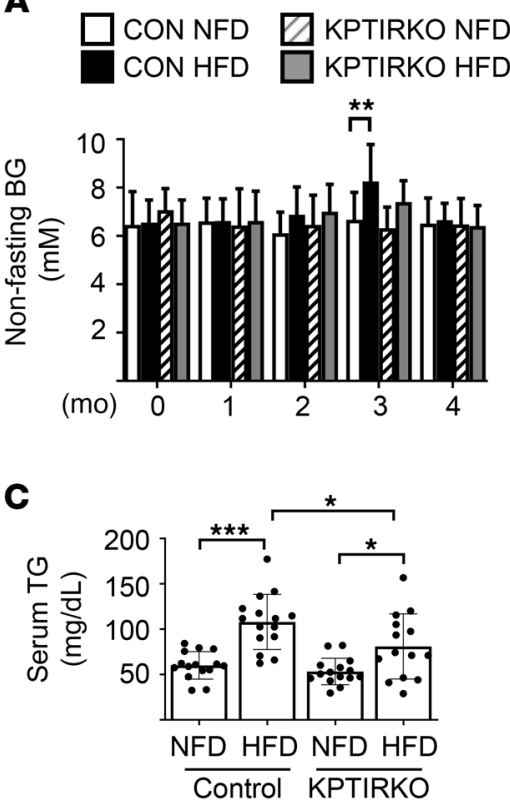

E

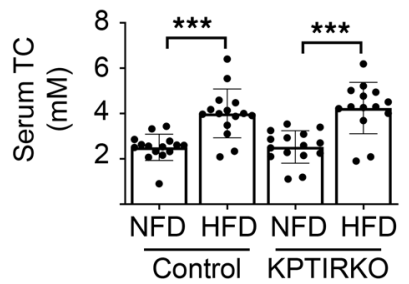

G

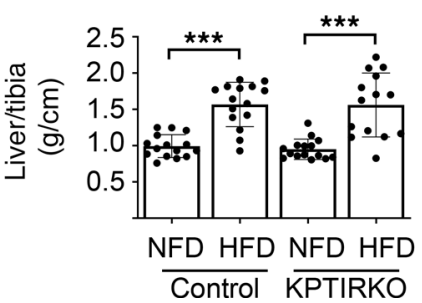

B
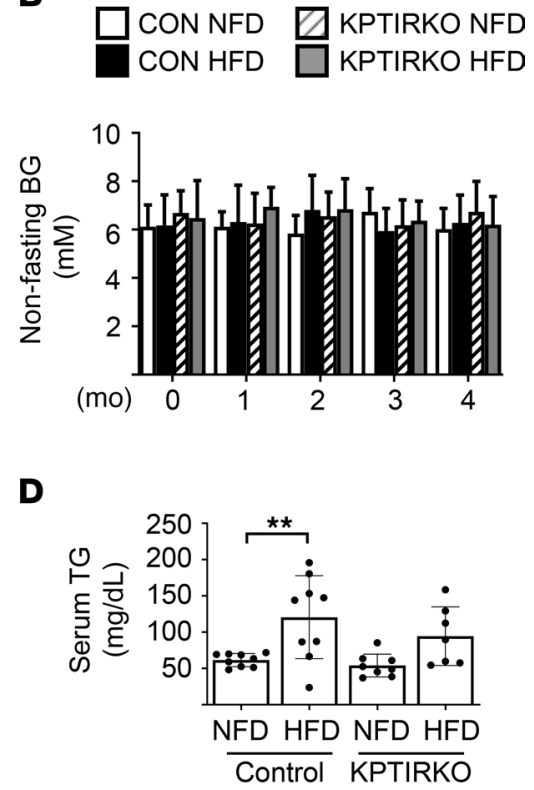

$\mathbf{F}$

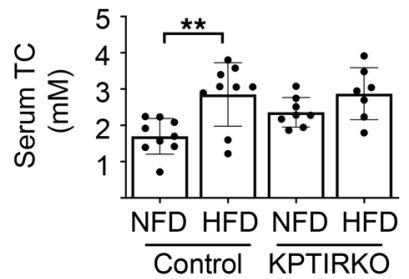

H

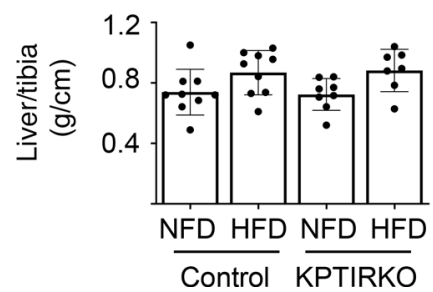

Figure 7. HFD regulation of metabolic parameters. (A and B) HFD did not affect nonfasting blood glucose in male $(n=$ 14-15 per group) or female mice of either genotype ( $n=7-9$ per group), except once at 3 months in male control mice. ( ${ }^{* *} P<0.01$ by 2 -way ANOVA and post hoc analysis using Tukey's multiple-comparisons test). (C and $\left.\mathbf{D}\right)$ Serum triglyceride (TC) level was increased by HFD in male control mice and increased to a lesser extent in KPTIRKO mice ( $n=14-15$ per group). It was also increased by HFD in female control mice but not in KPTIRKO mice ( $n=7-9$ per group). (E and F) HFD increased serum total cholesterol (TC) level in male control and KPTIRKO mice ( $n=14-15$ per group). The parameter was increased by HFD in female control mice; the change was not significant in female KPTIRKO mice ( $n=7-9$ per group). $(\mathbf{G}$ and $\mathbf{H})$ The liver-to-tibia ratio was increased by HFD in male control and KPTIRKO mice ( $n=14-15$ per group); HFD did not change the ratio in female mice of either genotype ( $n=7-9$ per group). $\left({ }^{*} P<0.05,{ }^{* *} P<0.01,{ }^{* *} P<0.001\right.$ by 1 -way ANOVA and post hoc analysis using Tukey's multiple-comparisons test). In the graphs, each dot represents 1 mouse. Data are presented as mean \pm SD.

cardiac deletion of IR ameliorates cardiac injury, demonstrating a direct role for IR in heart injury (26, 71). Of relevance to atherosclerosis, in insulin-resistant states, insulin's ability to activate the IR signaling pathway in vascular smooth muscle cells is impaired, leading to decreased nitric oxide generation and accelerated atherogenesis (70). Consistent with these observations, insulin augments endothelial expression of endothelin-1 (72, 73), PAI-1 (74), and vascular smooth muscle cell proliferation (75). Insulin has been shown to activate the MAP kinase pathway, despite the presence of insulin resistance in the 
Akt pathway, leading to oxidative stress in the wall of blood vessels of human subjects with coronary atherosclerosis (76). It is likely that hyperinsulinemia, when it occurs in the setting of other metabolic syndrome components, is especially injurious to the arterial wall (77). IGF-1 can signal through IGF$1 \mathrm{R}$, IR, or IGF-1R+IR hybrid receptors; however, IGF-1 is unlikely to be involved in our model because KPTIRKO mice resisted HFD-induced kidney injury.

Our data suggest a potentially novel mechanism of IR-mediated kidney injury in obesity. Decreased renal cortical production of $\mathrm{H}_{2} \mathrm{~S}$ was seen in HFD-fed control mice but not in KPTIRKO mice, suggesting that IR activation leads to $\mathrm{H}_{2} \mathrm{~S}$ deficiency. Recent studies have unraveled an important role for $\mathrm{H}_{2} \mathrm{~S}$ in defending kidney integrity in a variety of models of acute kidney injury and CKD (43). In aging-associated kidney injury, renal cortical IR activation with stimulation of the Akt/mTORC1 pathway is associated with $\mathrm{H}_{2} \mathrm{~S}$ deficiency (45). Administration of $\mathrm{H}_{2} \mathrm{~S}$ improves IR activation and the aforementioned indices of kidney injury (45). In the aging kidney, $\mathrm{H}_{2} \mathrm{~S}$ deficiency leads to IR activation, placing $\mathrm{H}_{2} \mathrm{~S}$ upstream of IR. Reduction in renal cortical expression of $\mathrm{H}_{2} \mathrm{~S}$-generating enzymes is also seen in rodent models of type 1 and 2 diabetes (47).

IR mediation of HFD-associated kidney injury is sex dependent. Compared with males, the female KPTIRKO mice had the same extent of IR deletion in the renal cortex, and, on HFD, gained comparable body weight and developed hypercholesterolemia. However, they sustained a milder form of HFD-induced kidney injury, as indicated by less albuminuria. On HFD, the female KPTIRKO mice did not develop elevated blood pressure and consequent heart hypertrophy; they did not show matrix protein accumulation or stimulation of signaling reactions related to protein synthesis. Renal cortical and urinary KIM-1 contents were unaffected in female mice on HFD. The ability of female mice to defend against HFD-induced kidney injury may be related to maintaining AMPK activity and $\mathrm{H}_{2} \mathrm{~S}$ generation. Impaired AMPK activity leads to kidney injury in diabetes and obesity, and conversely, stimulation of AMPK ameliorates kidney injury in those states $(9,37,78)$. Deficiency in $\mathrm{H}_{2} \mathrm{~S}$ generation is associated with kidney injury in diabetes and aging $(45,47,79)$, while administration of $\mathrm{H}_{2} \mathrm{~S}$ as NaHS ameliorates aging-associated kidney injury by stimulating AMPK activity and inhibiting IR activation associated Akt signaling related to protein synthesis (45). A similar salutary effect of $\mathrm{H}_{2} \mathrm{~S}$ has also been reported in rodents with diabetic kidney injury (80). Female mice maintained normal $\mathrm{H}_{2} \mathrm{~S}$ synthesis, despite reduction in CBS expression, indicating that compensation occurs at other sites in its synthetic pathway. Additionally, translating ribosome affinity purification analysis of proximal tubules has shown major differences in gene expression between male and female mice at baseline and following injury, suggesting a genetic mechanism for differential susceptibility (81). Many studies have shown that female sex is associated with lower incidence of hypertension, diabetic microangiopathy, progressive CKD, and death in CKD (82-85). Studies on mechanisms of sex differences in kidney injury suggest an injurious role for testosterone in males and a protective effect of estrogen in females (86-89).

A fascinating observation in the current study is that absence of IR in the proximal tubules reduces the extent of hypertriglyceridemia in HFD-induced obesity. This suggests a potential regulatory role for proximal tubular IR in the genesis of elevated triglycerides. More work is needed to understand if an axis exists between the kidney and other organs affecting uptake, synthesis, or degradation of triglycerides.

In conclusion, our data demonstrate a potentially new paradigm of kidney injury in HFD-induced obesity. Further exploration on renal IR-regulated cellular mechanisms involved in HFD-induced kidney injury is needed.

\section{Methods}

\section{Materials and sources}

We employed antibodies against the following: IR (catalog 3025, Cell Signaling Technology), Laminin $\gamma 1$ (catalog sc-5584, Santa Cruz Biotechnology), fibronectin (catalog ab2413, Abcam), type I collagen $\alpha 2$ (catalog 14695-1-AP, Proteintech), phospho-Ser9-GSK3 $\beta$ (catalog 9323, Cell Signaling Technology), GSK3 $\alpha / \beta$ (catalog sc-56913, Santa Cruz Biotechnology), phospho-Ser539-eIF2Be (catalog 44-530G, Thermo Fisher Scientific), eIF2B $\varepsilon$ (catalog 3595, Cell Signaling Technology), phospho-Ser2448-mTOR (catalog 2971, Cell Signaling Technology), mTOR (catalog 2972, Cell Signaling Technology), phospho-Thr389-p70S6 kinase (catalog 9205, Cell Signaling Technology), p70S6 kinase (catalog 9202, Cell Signaling Technology), CBS (catalog sc-67154, Santa Cruz Biotechnology), CSE (catalog sc-135203, Santa Cruz Biotechnology), IGF-1 receptor (catalog 3027, Cell 
Signaling Technology), phospho-Thr202/Tyr204-Erk (catalog 4377, Cell Signaling Technology), Erk (catalog 9102, Cell Signaling Technology), phospho-Ser473-Akt (catalog 9271, Cell Signaling Technology), Akt (catalog 9272, Cell Signaling Technology), nephrin (catalog ab58968, Abcam), and actin (catalog A2066, MilliporeSigma). Commercial ELISA kits were used for measuring phospho-Tyr-1150/1151-IR (catalog 7082, Cell Signaling Technology) and phospho-Ser79-ACC (catalog 7986, Cell Signaling Technology).

\section{Animals}

Generation of KPTIRKO mice. Sodium-dependent glucose transporter 2 (Sglt2) is located predominantly in the S1 segment of the proximal tubules (23) and governs glucose reabsorption; thus, the Sglt2 promoter is specific for proximal tubule targeting (90). We confirmed the specificity of Sglt2 for the proximal tubules by crossing Sglt2 Cre mice with Rosa26 reporter mice designed to express LacZ; in the hybrid mice, $\beta$-galactosidase activity detected by X-gal staining was seen only in the proximal tubules of the kidney and not in the other parts of nephron or other organs (Supplemental Figure 7). We generated KPTIRKO mice by crossing Sglt2 Cre mice (provided by I. Rubera and M. Tauc, LP2M CNRS/Université Côte d'Azur laboratory, Nice, France; the strain is now archived and distributed by the European Mouse Mutant Archive, https://www.infrafrontier.eu/resources-and-services/access-emma-mouse-resources_with Ir-loxp mice, in which exon 4 of the Ir $\beta$ chain is floxed (provided in-house; catalog 006955 , The Jackson Laboratory). Ir-loxp Cre-negative littermates were used as the control mice (Supplemental Figure 7). Initially, 8 generations of back crossing were done to bring Ir-Loxp mice to the C57BL6 genotype. At the beginning of Cre-Lox crossing both mice were on C57BL6 background.

We used a model of HFD-induced obesity in which hyperinsulinemia occurs in association with normal blood glucose levels. We randomized 5- to 8-month-old male and female control and KPTIRKO mice to receive HFD or NFD for 4 months. In the HFD, $60 \%$ of the calories are derived from fat (D12492, Research Diets Inc.), whereas $10 \%$ of the calories are fat derived in the NFD (D12450J, Research Diets). Three separate batches (male) and 2 separate batches (female) of mice were studied (male: control NFD, $n=15$; control HFD, $n=15$; KPTIRKO NFD, $n=15$; KPTIRKO HFD, $n=14$; and female: control NFD, $n=9$; control HFD, $n=9$; KPTIRKO NFD, $n=8$; KPTIRKO HFD, $n=7$ ).

Genotyping. Lysis buffer (50 mM KCl, $10 \mathrm{mM}$ Tris- $\mathrm{HCl} \mathrm{pH} 8.3,2.5 \mathrm{mM} \mathrm{MgCl}, 0.1 \mathrm{mg} / \mathrm{mL}$ gelatin, 0.45\% IGEPAL-CA630, 0.45\% Tween 20, and $60 \mu \mathrm{g} / \mathrm{mL}$ Proteinase K (Thermo Fisher Scientific) was used to prepare DNA from the tails of mice. Cre-recombinase gene was amplified using the following primers: forward, 5'-AGGTTCGTTCACTCATGGA-3', and reverse, 5'-TCGACCAGTTTAGTTACCC-3'. We used the following primers (Jackson Laboratory genotyping protocol) to amplify the region in the targeted Ir allele spanning the loxp sites: forward, 5'-GGGGCAGTGAGTATTTTGGA-3', and reverse, 5'-TGGCCGTGAAAGTTAAGAGG-3'. The band size of the mutant allele was $145 \mathrm{bp}$, and the band size of the wild-type recombined allele had a size of $105 \mathrm{bp}$ (Supplemental Figure 7).

$B P$ measurement. The tail cuff method was employed to measure blood pressure as previously described (22).

Immunoblotting. SDS PAGE analysis of proteins and protocols for incubation with respective antibodies have been described previously $(45,47,79,91)$.

Urinary ACR ratio and blood glucose measurement. We employed analytical kits to measure albumin (catalog E101 and E90-134, Bethyl Laboratories Inc.) and creatinine (catalog ADI-907-030A, Enzo Life Sciences Inc.). Blood glucose concentration was measured by a glucometer (Ascensia Diabetes Care US Inc.).

IR tyrosine phosphorylation and phosphorylation of ACC. IR tyrosine phosphorylation and phosphorylation of ACC were measured by using ELISA kits according to the instructions provided by the manufacturers.

Insulin measurement. Mouse serum insulin levels were determined using the mouse metabolic hormone panel according to the manufacturer's instructions (Milliplex MMHMAG-44K, MilliporeSigma). Assays were performed on a MAGPIX Multiplexing instrument, and data were analyzed using XPONENT 4.2 software.

\section{Statistics}

Data were expressed as mean $\pm \mathrm{SD}$. Analyses between 2 groups were performed using the 2-tailed $t$ test using GraphPad Prism 8. Data were considered statistically significant at $P<0.05$. Statistical comparisons between multiple groups were performed by 1-way or 2-way ANOVA, and post hoc analysis was done using Tukey's multiple-comparisons test, employing GraphPad Prism 8 software. $P<0.05$ was considered significant. 


\section{Study approval}

The Institutional Animal Care and Use Committees of the University of Texas Health and South Texas Veterans Health Care System approved the animal experiments.

\section{Author contributions}

BSK supervised the project in its conception, design, and data interpretation and wrote the manuscript. HJL and MMM conducted experiments, with the aid of SBO, AD, and CSR. LN and TB performed insulin assays. CRK, IR and MT provided genetically modified mice. SEH helped with the generation of KPTIRKO mice. MMM, LN, GGC, DF, MAV, KS, CRK, and RAD participated in data interpretation and writing of the manuscript. All authors provided input and approved the manuscript.

\section{Acknowledgments}

This work was supported by the Department of Veterans Affairs Biomedical Laboratory Research and Development Service Merit Review Award (I01 BX001340 to BSK and KS). GGC is supported by the VA Merit Review Award (2I01 BX000926) and Research Career Scientist Award (IK6BX00361). KS is supported by the VA Merit Review Award (1I01BX003234).

Address correspondence to: Balakuntalam S. Kasinath, Division of Nephrology, Department of Medicine, MC7882, University of Texas Health at San Antonio, 7703 Floyd Curl Drive, San Antonio, Texas 78229, USA. Phone: 210.326.0929; Email: kasinath@uthsca.edu.

DF's present address is: Genomic Institute of the Novartis Research Foundation, La Jolla, California.

1. Finucane MM, et al. National, regional, and global trends in body-mass index since 1980: systematic analysis of health examination surveys and epidemiological studies with 960 country-years and 9·1 million participants. Lancet. 2011;377(9765):557-567.

2. Kramer H, et al. Obesity and prevalent and incident CKD: the hypertension detection and follow-up program. Am J Kidney Dis. 2005;46(4):587-594

3. Shen WW, et al. Obesity-related glomerulopathy: body mass index and proteinuria. Clin J Am Soc Nephrol. 2010;5(8):1401-1409.

4. Hales CM, et al. Prevalence of obesity among adults and youth: United States, 2015-2016. https://www.cdc.gov/nchs/data/ databriefs/db288.pdf. October 2017. Accessed January 25, 2021.

5. Ward ZJ, et al. Projected US state-level prevalence of adult obesity and severe obesity. N Engl J Med. 2019;381(25):2440-2450

6. Choung HG, et al. The spectrum of kidney biopsy findings in patients with morbid obesity. Kidney Int. 2019;95(3):647-654.

7. Hashimoto Y, et al. Metabolically healthy obesity and risk of incident CKD. Clin J Am Soc Nephrol. 2015;10(4):578-583.

8. Decleves AE, et al. AMPK mediates the initiation of kidney disease induced by a high-fat diet. J Am Soc Nephrol. 2011;22(10):1846-1855.

9. Decleves AE, et al. Regulation of lipid accumulation by AMP-activated kinase [corrected] in high fat diet-induced kidney injury. Kidney Int. 2014;85:611-623

10. Sharma K, et al. Adiponectin regulates albuminuria and podocyte function in mice. J Clin Invest. 2008;118(5):1645-1656.

11. Ecelbarger CM, et al. The effect of chronic candesartan therapy on the metabolic profile and renal tissue cytokine levels in the obese Zucker rat. Mediators Inflamm. 2010;2010:841343

12. de Jong PE, et al. Obesity and target organ damage: the kidney. Int J Obes Relat Metab Disord. 2002;26(suppl 4):S21-24.

13. Wu Y, et al. Obesity-related glomerulopathy: insights from gene expression profiles of the glomeruli derived from renal biopsy samples. Endocrinology. 2006;147(1):44-50.

14. Griffin KA, et al. Adverse renal consequences of obesity. Am J Physiol Renal Physiol. 2008;294(4):F685-F696.

15. Riazi S, et al. Abundance of the Na-K-2Cl cotransporter NKCC2 is increased by high-fat feeding in Fischer $344 \mathrm{X}$ Brown Norway (F1) rats. Am J Physiol Renal Physiol. 2009;296(4):F762-F770.

16. Davies M, et al. Novel mechanisms of $\mathrm{Na}^{+}$retention in obesity: phosphorylation of NKCC2 and regulation of SPAK/OSR1 by AMPK. Am J Physiol Renal Physiol. 2014;307(1):F96-F106.

17. Ha TS, et al. Regulation of renal laminin in mice with type II diabetes. J Am Soc Nephrol. 1999;10(9):1931-1939.

18. Feliers D, et al. Activation of renal signaling pathways in db/db mice with type 2 diabetes. Kidney Int. 2001;60(2):495-504

19. Mariappan MM, et al. High glucose, high insulin, and their combination rapidly induce laminin-beta1 synthesis by regulation of mRNA translation in renal epithelial cells. Diabetes. 2007;56(2):476-485.

20. Sataranatarajan K, et al. Regulation of elongation phase of mRNA translation in diabetic nephropathy: amelioration by rapamycin. Am J Pathol. 2007;171(6):1733-1742.

21. Bhandari BK, et al. Insulin regulation of protein translation repressor 4E-BP1, an eIF4E-binding protein, in renal epithelial cells. Kidney Int. 2001;59(3):866-875

22. Mariappan MM, et al. Combined acute hyperglycemic and hyperinsulinemic clamp induced profibrotic and proinflammatory responses in the kidney. Am J Physiol Cell Physiol. 2014;306(3):C202-C211.

23. Kanai $\mathrm{Y}$, et al. The human kidney low affinity $\mathrm{Na}+$ /glucose cotransporter SGLT2. Delineation of the major renal reabsorptive mechanism for D-glucose. J Clin Invest. 1994;93(1):397-404.

24. Zhai XY, et al. Digital three-dimensional reconstruction and ultrastructure of the mouse proximal tubule. J Am Soc Nephrol. 
2003;14(3):611-619

25. Han WK, et al. Kidney injury molecule-1 (KIM-1): a novel biomarker for human renal proximal tubule injury. Kidney Int. 2002;62(KIM-1):237-244.

26. Wang $Q$, et al. Inhibiting insulin-mediated $\beta 2$-adrenergic receptor activation prevents diabetes-associated cardiac dysfunction. Circulation. 2017;135(1):73-88.

27. White MF, et al. A cascade of tyrosine autophosphorylation in the beta-subunit activates the phosphotransferase of the insulin receptor. J Biol Chem. 1988;263(6):2969-2980.

28. Kasinath BS, et al. mRNA translation: unexplored territory in renal science. J Am Soc Nephrol. 2006;17(12):3281-3292.

29. Kasinath BS, et al. Regulation of mRNA translation in renal physiology and disease. Am J Physiol Renal Physiol. 2009;297(5):F1153-F1165.

30. Cross DA, et al. Inhibition of glycogen synthase kinase-3 by insulin mediated by protein kinase B. Nature. 1995;378(6559):785-789

31. Leger B, et al. Akt signalling through GSK-3beta, mTOR and Foxo1 is involved in human skeletal muscle hypertrophy and atrophy. J Physiol. 2006;576(Pt 3):923-933.

32. Mariappan MM, et al. Glycogen synthase kinase $3 \beta$ is a novel regulator of high glucose- and high insulin-induced extracellular matrix protein synthesis in renal proximal tubular epithelial cells. J Biol Chem. 2008;283(45):30566-30575.

33. Mariappan MM, et al. Activation of glycogen synthase kinase $3 \beta$ ameliorates diabetes-induced kidney injury. $J$ Biol Chem. 2014;289(51):35363-35375.

34. Doble BW, Woodgett JR. GSK-3: tricks of the trade for a multi-tasking kinase. J Cell Sci. 2003;116(Pt 7):1175-1186

35. Wang X, Proud CG. A novel mechanism for the control of translation initiation by amino acids, mediated by phosphorylation of eukaryotic initiation factor 2B. Mol Cell Biol. 2008;28(5):1429-1442.

36. Holz MK, et al. mTOR and S6K1 mediate assembly of the translation preinitiation complex through dynamic protein interchange and ordered phosphorylation events. Cell. 2005;123(4):569-580.

37. Lee MJ, et al. A role for AMP-activated protein kinase in diabetes-induced renal hypertrophy. Am J Physiol Renal Physiol. 2007;292(2):F617-F627.

38. Eid AA, et al. AMP-activated protein kinase (AMPK) negatively regulates Nox4-dependent activation of p53 and epithelial cell apoptosis in diabetes. J Biol Chem. 2010;285(48):37503-37512.

39. Decleves AE, et al. AMP-activated protein kinase activation ameliorates eicosanoid dysregulation in high-fat-induced kidney disease in mice. J Lipid Res. 2019;60(5):937-952.

40. Hawley SA, et al. Phosphorylation by Akt within the ST loop of AMPK- $\alpha 1$ down-regulates its activation in tumour cells. Biochem J. 2014;459(2):275-287.

41. Xia M, et al. Production and actions of hydrogen sulfide, a novel gaseous bioactive substance, in the kidneys. J Pharmacol Exp Ther. 2009;329(3):1056-1062.

42. Feliers D, et al. Hydrogen sulfide in renal physiology and disease. Antioxid Redox Signal. 2016;25(13):720-731

43. Kasinath BS, et al. Hydrogen sulfide as a regulatory factor in kidney health and disease. Biochem Pharmacol. 2018;149:29-41.

44. Shibuya N, et al. A novel pathway for the production of hydrogen sulfide from D-cysteine in mammalian cells. Nat Commun. 2013;4:1366.

45. Lee HJ, et al. Hydrogen sulfide ameliorates aging-associated changes in the kidney. Geroscience. 2018;40(2):163-176.

46. Lee HJ, et al. Marmoset as a model to study kidney changes associated with aging. J Gerontol A Biol Sci Med Sci. 2019;74(3):315-324.

47. Lee HJ, et al. Hydrogen sulfide inhibits high glucose-induced matrix protein synthesis by activating AMP-activated protein kinase in renal epithelial cells. J Biol Chem. 2012;287(7):4451-4461.

48. Corkey BE. Banting lecture 2011: hyperinsulinemia: cause or consequence? Diabetes. 2012;61(1):4-13.

49. Czech MP. Insulin action and resistance in obesity and type 2 diabetes. Nat Med. 2017;23(7):804-814.

50. Nakamura R, et al. Insulin binding sites in various segments of the rabbit nephron. J Clin Invest. 1983;72(1):388-392.

51. Hale LJ, Coward RJ. The insulin receptor and the kidney. Curr Opin Nephrol Hypertens. 2013;22(1):100-106.

52. Welsh GI, et al. Insulin signaling to the glomerular podocyte is critical for normal kidney function. Cell Metab. 2010;12(4):329-340.

53. Tiwari S, et al. Deletion of the insulin receptor in the proximal tubule promotes hyperglycemia. J Am Soc Nephrol. 2013;24(8):1209-1214

54. Murtha MJ, et al. Insulin receptor signaling regulates renal collecting duct and intercalated cell antibacterial defenses. $J$ Clin Invest. 2018;128(12):5634-5646.

55. DeFronzo RA, et al. The effect of insulin on renal handling of sodium, potassium, calcium, and phosphate in man. J Clin Invest 1975;55(4):845-855.

56. Ecelbarger CM. Refining insulin signaling in the proximal tubule at the level of the substrate. Kidney Int. 2020;97(2):256-258.

57. Bryniarski MA, et al. Increased megalin expression in early type 2 diabetes: role of insulin-signaling pathways. Am J Physiol Renal Physiol. 2018;315(5):F1191-F1207.

58. Alsahli M, Gerich JE. Renal glucose metabolism in normal physiological conditions and in diabetes. Diabetes Res Clin Pract. 2017;133:1-9.

59. Tiwari S, et al. Impaired sodium excretion and increased blood pressure in mice with targeted deletion of renal epithelial insulin receptor. Proc Natl Acad Sci U S A. 2008;105(17):6469-6474.

60. Nakamura $M$, et al. Insulin promotes sodium transport but suppresses gluconeogenesis via distinct cellular pathways in human and rat renal proximal tubules. Kidney Int. 2020;97(2):316-326.

61. Nakamura M, et al. Preserved $\mathrm{Na} / \mathrm{HCO} 3$ cotransporter sensitivity to insulin may promote hypertension in metabolic syndrome Kidney Int. 2015;87(3):535-542.

62. $\mathrm{Li} \mathrm{L}$, et al. Reduced $\mathrm{ENaC}$ activity and blood pressure in mice with genetic knockout of the insulin receptor in the renal collecting duct. Am J Physiol Renal Physiol. 2013;304(3):F279-F288.

63. Lu M, et al. mTOR complex-2 activates ENaC by phosphorylating SGK1. J Am Soc Nephrol. 2010;21(5):811-818

64. Chavez-Canales M, et al. Insulin increases the functional activity of the renal NaCl cotransporter. J Hypertens. 2013;31(2):303-311.

65. Giordano M, et al. Differential responsiveness of protein synthesis and degradation to amino acid availability in humans. Diabetes. $1996 ; 45(4): 393-399$ 
66. Kondo T, et al. Knockout of insulin and IGF-1 receptors on vascular endothelial cells protects against retinal neovascularization. J Clin Invest. 2003;111(12):1835-1842.

67. Kondo T, Kahn CR. Altered insulin signaling in retinal tissue in diabetic states. J Biol Chem. 2004;279(36):37997-38006.

68. Despres JP, et al. Hyperinsulinemia as an independent risk factor for ischemic heart disease. N Engl J Med. 1996;334(15):952-957.

69. Pyorala M, et al. Hyperinsulinemia predicts coronary heart disease risk in healthy middle-aged men: the 22-year follow-up results of the Helsinki Policemen Study. Circulation. 1998;98(5):398-404.

70. DeFronzo RA. Insulin resistance, lipotoxicity, type 2 diabetes and atherosclerosis: the missing links. The Claude Bernard Lecture 2009. Diabetologia. 2010;53(7):1270-1287.

71. Shimizu I, et al. Excessive cardiac insulin signaling exacerbates systolic dysfunction induced by pressure overload in rodents. J Clin Invest. 2010;120(5):1506-1514.

72. Oliver FJ, et al. Stimulation of endothelin-1 gene expression by insulin in endothelial cells. J Biol Chem. 1991;266(34):23251-23256

73. Cardillo C, et al. Insulin stimulates both endothelin and nitric oxide activity in the human forearm. Circulation. 1999;100(8):820-825.

74. Nordt TK, et al. Augmentation of arterial endothelial cell expression of the plasminogen activator inhibitor type-1 (PAI-1) gene by proinsulin and insulin in vivo. J Mol Cell Cardiol. 1998;30(8):1535-1543.

75. Banskota NK, et al. Insulin, insulin-like growth factor I and platelet-derived growth factor interact additively in the induction of the protooncogene c-myc and cellular proliferation in cultured bovine aortic smooth muscle cells. Mol Endocrinol. 1989;3(8):1183-1190.

76. Akoumianakis I, et al. Insulin-induced vascular redox dysregulation in human atherosclerosis is ameliorated by dipeptidyl peptidase 4 inhibition. Sci Transl Med. 2020;12(541):eaav8824.

77. Rask-Madsen C, et al. Hyperinsulinemia does not change atherosclerosis development in apolipoprotein E null mice. Arterioscler Thromb Vasc Biol. 2012;32(5):1124-1131.

78. Dugan LL, et al. AMPK dysregulation promotes diabetes-related reduction of superoxide and mitochondrial function. $J$ Clin Invest. 2013;123(11):4888-4899.

79. Lee HJ, et al. Hydrogen sulfide inhibits high glucose-induced NADPH oxidase 4 expression and matrix increase by recruiting inducible nitric oxide synthase in kidney proximal tubular epithelial cells. J Biol Chem. 2017;292(14):5665-5675.

80. Zhou X, et al. Hydrogen sulfide alleviates diabetic nephropathy in a streptozotocin-induced diabetic rat model. J Biol Chem. 2014;289(42):28827-28834.

81. Wu H, et al. Proximal tubule translational profiling during kidney fibrosis reveals proinflammatory and long noncoding RNA expression patterns with sexual dimorphism. J Am Soc Nephrol. 2020;31(1):23-38.

82. Yu M, et al. Clinical implication of metabolic syndrome on chronic kidney disease depends on gender and menopausal status: results from the Korean National Health and Nutrition Examination Survey. Nephrol Dial Transplant. 2010;25(2):469-477.

83. Benjamin EJ, et al. Heart disease and stroke statistics-2017 update: a report from the American Heart Association. Circulation. 2017;135(10):e146-e603.

84. Maric-Bilkan C. Sex differences in micro- and macro-vascular complications of diabetes mellitus. Clin Sci (Lond). 2017;131(9):833-846.

85. Ricardo AC, et al. Sex-related disparities in CKD progression. J Am Soc Nephrol. 2019;30(1):137-146.

86. Zhang MZ, et al. The role of the EGF receptor in sex differences in kidney injury. J Am Soc Nephrol. 2019;30(9):1659-1673.

87. Inada A, et al. Adjusting the 17ß-estradiol-to-androgen ratio ameliorates diabetic nephropathy. J Am Soc Nephrol. 2016;27(10):3035-3050.

88. Seppi T, et al. Sex differences in renal proximal tubular cell homeostasis. J Am Soc Nephrol. 2016;27(10):3051-3062.

89. Garovic VD, August P. Sex differences and renal protection: keeping in touch with your feminine side. J Am Soc Nephrol. 2016;27(10):2921-2924.

90. Rubera I, et al. Specific Cre/Lox recombination in the mouse proximal tubule. J Am Soc Nephrol. 2004;15(8):2050-2056.

91. Lee HJ, et al. Tadalafil integrates nitric oxide-hydrogen sulfide signaling to inhibit high glucose-induced matrix protein synthesis in podocytes. J Biol Chem. 2015;290(19):12014-12026. 